\title{
Scaling predictions for radii of weakly bound triatomic molecules
}

\author{
M. T. Yamashita \\ Laboratório do Acelerador Linear, Instituto de Física, Universidade de São Paulo, Caixa Postal 66318, CEP 05315-970, \\ São Paulo, Brazil \\ R. S. Marques de Carvalho and Lauro Tomio \\ Instituto de Física Teórica, Universidade Estadual Paulista, 01405-900, São Paulo, Brazil \\ T. Frederico \\ Departamento de Física, Instituto Tecnológico de Aeronáutica, Centro Técnico Aeroespacial, 12228-900, São José dos Campos, Brazil
}

(Received 8 March 2003; published 16 July 2003)

\begin{abstract}
The mean-square radii of the molecules ${ }^{4} \mathrm{He}_{3},{ }^{4} \mathrm{He}_{2}-{ }^{6} \mathrm{Li},{ }^{4} \mathrm{He}_{2}-{ }^{7} \mathrm{Li}$, and ${ }^{4} \mathrm{He}_{2}-{ }^{23} \mathrm{Na}$ are calculated using a three-body model with contact interactions. They are obtained from a universal scaling function calculated within a renormalized scheme for three particles interacting through pairwise Dirac- $\delta$ interaction. The rootmean-square distance between two atoms of mass $m_{A}$ in a triatomic molecule are estimated to be of the order of $\mathcal{C} \sqrt{\hbar^{2} /\left[m_{A}\left(E_{3}-E_{2}\right)\right]}$, where $E_{2}$ is the dimer and $E_{3}$ is the trimer binding energies, and $\mathcal{C}$ is a constant (varying from $\sim 0.6$ to $\sim 1$ ), which depends on the ratio between $E_{2}$ and $E_{3}$. Considering previous estimates for the trimer energies, we also predict the sizes of rubidium and sodium trimers in atomic traps.
\end{abstract}

DOI: 10.1103/PhysRevA.68.012506

PACS number(s): 31.50.-x, 34.10.+x, 36.40.-c

\section{INTRODUCTION}

Weakly bound molecules are large size quantum systems in which the atoms have an appreciable probability to be found much beyond the interaction range, and at the same time tiny changes in the potential parameters can produce huge effects in the properties of these states [1]. The best illustration of such systems is the experimentally found ${ }^{4} \mathrm{He}_{2}$ dimer [2], with $\sqrt{\left\langle r^{2}\right\rangle}=52 \pm 4 \AA$ and binding energy $E_{2}$ $=1.1+0.3 /-0.2 \mathrm{mK}[3]$. Other examples of weakly bound molecules are found through the experimental realization of Bose-Einstein condensation (BEC) [4], where the possibility to change the effective scattering length of the low-energy atom-atom interaction in the trap to large positive values by using an external magnetic field $[4,5]$ can produce very large dimers. In fact, weakly bound molecules in ultracold atomic traps were reported in Ref. [6]. The binding energy of the ${ }^{87} \mathrm{Rb}_{2}$ dimer formed in a Bose-Einstein condensate was measured with unprecedented accuracy [7]. Ultracold $\mathrm{Na}_{2}$ molecules have also been formed through photoassociation [8]. One should note that in the limit of an infinite atom-atom scattering length tuned by the Feshbach resonances in the trap, in principle the Efimov condition [9] can be achieved, in which an infinite number of weakly bound trimers exist. The formation of weakly bound trimers in ultracold atomic systems have not been reported till now, but recently the recombination coefficient rate was used to predict trimer binding energies of some specific atomic species that are being studied in atomic traps [10].

Theoretically it is possible to exist weakly bound molecules of zero-angular momentum states in triatomic systems, as for example, in the extensively studied ${ }^{4} \mathrm{He}$ trimer system (see, e.g., Ref. [11] and therein). These molecules are special due to the large spatial size, which spreads out much beyond the potential range $[11,12]$. In such trimer, the calculations of the mean-square distance of each ${ }^{4} \mathrm{He}$ atom to the corresponding center of mass (c.m.) have been performed for the ground and excited states [11,12], and also for the meansquare interatomic distance [11]. These sizes are of the order of 5-10 $\AA$ for the ground state of the ${ }^{4} \mathrm{He}_{3}$ molecule, and of about 50-90 ^ for the excited Efimov state [11]. Therefore, the system heals through the regions that are well outside of the potential range, in which the wave function is essentially a solution of the free Schrödinger equation, and where the physical properties of the bound system is defined by a few physical scales. For example, the dimensionless product of the mean-square interatom distance with the separation energy of one atom from the trimer is not far from the unity [11] in the ground and also in the excited states, despite the large difference between such energies $E_{3}^{(0)} / E_{3}^{(1)} \approx 50\left(E_{3}^{(n)}\right.$ is the binding energy of the $n$th trimer state). So, as already discussed in Refs. [13-16], we should note that quite naturally the binding energy is the scale that dominates the physics of the trimer. One should remember as well that the collapse of the three-body system in the limit of a zero-range force [17] makes the three-body energy one of the scales of the system, beyond the two-body energy [13].

The calculation of the low-energy properties of the threebody system can be performed with a renormalization scheme applied to three-body equations with $s$ wave zerorange pairwise potential $[13,18]$. In this approach, one can fix the three-body ground-state (the three-body physical scale) and the two-body scattering lengths [13]. Consequently, all the detailed information about the short-range force, beyond the low-energy two-body observables, are retained in only one three-body physical information in the limit of zero-range interaction.

In the present work, we first study the mean-square distances of one atom to the c.m. system and between two atoms in the ground and excited states of triatomic molecules of type ${ }^{4} \mathrm{He}_{2}-X$, where $X \equiv{ }^{4} \mathrm{He},{ }^{6} \mathrm{Li},{ }^{7} \mathrm{Li}$, and ${ }^{23} \mathrm{Na}$. Next, using trimer energies derived from the recombination 
coefficient rates [10], we make estimates of the corresponding sizes of rubidium and sodium trimers. We introduce and calculate scaling functions that describe the different radii as functions of the physical scales of the triatomic system obtained in the limit of a zero-range interaction. In this way, we are generalizing the concept of scaling function, which was previously introduced in Refs. [15] and [18] to study the behavior of bound and excited virtual Efimov states [9] in terms of triatomic physical scales.

The scaling function depends only on dimensionless ratios of the binding energies of two and three atoms, and the ratio of masses of the different atoms. In that sense our conclusions apply equally well to any other low-energy triatomic system. The validity condition for the scaling relations is that the interaction range must be small compared to particle distances, which is the case for weakly bound three-body systems.

The paper is organized as follows. In Sec. II, we present the Faddeev equations for the spectator functions for a triatomic system with two equal particles $\alpha$ and a third one $\beta$, and the form factors from which the different mean-square radii are obtained. Also in this section we discuss the generalization of the scaling function defined in Refs. $[15,18]$ to describe the different radii. In Sec. III, we present our numerical results for the mean-square distances of one atom with respect to the c.m. system and between two atoms in the ground and excited states of triatomic molecules. Our conclusions are summarized in Sec. IV.

\section{RENORMALIZED THREE-BODY MODEL AND FORM FACTORS}

In this section, we introduce the generalization of the scaling function defined in Refs. [15] and [18], to be used to obtain the different radii. We write down the coupled renormalized equations for the spectator functions and the expressions for the form factors, which allow the calculation of the different mean-square distances.

\section{A. Subtracted Faddeev equations}

Throughout this paper we use units such that $\hbar=m_{\alpha}$ $=1$. For $\alpha={ }^{4} \mathrm{He}, \hbar^{2} / m_{4} \mathrm{He}_{\mathrm{e}}=12.12 \mathrm{~K}^{2}$. After partial wave projection, the $s$-wave coupled subtracted integral equations, for two identical particles $\alpha$ and a third one $\beta$, are given by

$$
\begin{aligned}
& \chi_{\alpha \alpha}(y)=2 \tau_{\alpha \alpha}\left(y ; \epsilon_{3}\right) \int_{0}^{\infty} d x \frac{x}{y} G_{1}\left(y, x ; \epsilon_{3}\right) \chi_{\alpha \beta}(x), \\
& \chi_{\alpha \beta}(y)=\tau_{\alpha \beta}\left(y ; \epsilon_{3}\right) \int_{0}^{\infty} d x \frac{x}{y}\left[G_{1}\left(x, y ; \epsilon_{3}\right) \chi_{\alpha \alpha}(x)\right. \\
& \left.+A G_{2}\left(y, x ; \epsilon_{3}\right) \chi_{\alpha \beta}(x)\right], \\
& \tau_{\alpha \alpha}\left(y ; \epsilon_{3}\right) \equiv \frac{1}{\pi}\left[\sqrt{\epsilon_{3}+\frac{A+2}{4 A} y^{2}} \mp \sqrt{\epsilon_{\alpha \alpha}}\right]^{-1},
\end{aligned}
$$

$$
\begin{gathered}
\tau_{\alpha \beta}\left(y ; \epsilon_{3}\right) \equiv \frac{1}{\pi}\left(\frac{A+1}{2 A}\right)^{3 / 2}\left[\sqrt{\epsilon_{3}+\frac{A+2}{2(A+1)} y^{2}} \mp \sqrt{\epsilon_{\alpha \beta}}\right]^{-1}, \\
G_{1}\left(y, x ; \epsilon_{3}\right) \equiv \log \frac{2 A\left(\epsilon_{3}+x^{2}+x y\right)+y^{2}(A+1)}{2 A\left(\epsilon_{3}+x^{2}-x y\right)+y^{2}(A+1)} \\
-\log \frac{2 A\left(1+x^{2}+x y\right)+y^{2}(A+1)}{2 A\left(1+x^{2}-x y\right)+y^{2}(A+1)}, \\
G_{2}\left(y, x ; \epsilon_{3}\right) \equiv \\
\log \frac{2\left(A \epsilon_{3}+x y\right)+\left(y^{2}+x^{2}\right)(A+1)}{2\left(A \epsilon_{3}-x y\right)+\left(y^{2}+x^{2}\right)(A+1)} \\
-\log \frac{2(A+x y)+\left(y^{2}+x^{2}\right)(A+1)}{2(A-x y)+\left(y^{2}+x^{2}\right)(A+1)} .
\end{gathered}
$$

The mass number $A$ is given by the ratio $m_{\beta} / m_{\alpha}$. The plus and minus signs in Eq. (3) and (4) refer to virtual and bound two-body subsystems, respectively.

In the present context that we have three particle systems with two identical ones, it is worthwhile to call the attention to two particular definitions of three-body quantum halo states: the Borromean states [19], where all the two-body subsystems are virtual $(\alpha-\alpha-\beta)$, and the tango states [20], where the $\alpha-\beta$ subsystems are virtual and $\alpha \alpha$ is bound $(\alpha \alpha-\beta)$. Note that the virtual pair of particles is denoted with a dash between the symbols. The Borromean case corresponds to positive signs in front of the square-root energy of the subsystems in both Eqs. (3) and (4), implying in the weakest attractive kernel of Eqs. (1) and (2) among all the possibilities of signs in the two-body scattering amplitude, and, for the tango three-body system, we have negative sign only in front of $\sqrt{\epsilon_{\alpha \alpha}}$ in Eq. (3), with positive sign in front of $\sqrt{\epsilon_{\alpha \beta}}$ in Eq. (4). So, a more effective attraction occurs in a tango state than in a Borromean case. Of course, if all the two-body subsystems are bound, the effective attraction is maximized, and, if all such subsystems are unbound (virtual), the effective attraction is minimized.

One can extend the classification scheme of three-body quantum halo states of the type $\alpha \alpha \beta$ [21], considering the four possibilities, for increasing values of the magnitude of the effective attraction in Eqs. (1) and (2). The weakest attractive situation corresponds to the previous defined Borromean-type (only virtual subsystems) $(\alpha-\alpha-\beta)$. The tango situation $(\alpha \alpha-\beta)$ is followed by a three-body system with $\alpha-\alpha$ virtual and $\alpha \beta$ bound, which we represent by $(\alpha \beta \alpha)$ halo system. Three-body system with the strongest effective attraction has all the subsystems bound and is represented by $(\alpha \alpha \beta)$.

We solve Eqs. (1)-(6) in units such that the three-body subtraction point $\mu_{(3)}$ is equal to 1 [18]. The corresponding dimensionless quantities are $\epsilon_{3} \equiv E_{3} / \mu_{(3)}^{2}, \epsilon_{\alpha \alpha} \equiv E_{\alpha \alpha} / \mu_{(3)}^{2}$, $\epsilon_{\alpha \beta} \equiv E_{\alpha \beta} / \mu_{(3)}^{2}$. The three-body physical quantities can be written in terms of the three-body binding energy $E_{3}$ when first the value of $\mu_{(3)}^{2}$ is determined from the known value of $E_{3}$. Therefore, the results for the renormalized model appear 
when the subtraction point energy is written as a function of $E_{3}$ and, consequently, the three-body quantities naturally scale with $E_{3}$. Finally, the scaling functions are obtained when the dimensionless product of physical quantities are written as a function of the ratios between two-body energies and $E_{3}$.

\section{B. Scaling functions for the radii}

The existence of a three-body scale implies in the lowenergy universality found in three-body systems, or correlations between three-body observables $[13,22]$. In the scaling limit $[14,18]$, one has

$$
\begin{aligned}
\mathcal{O}\left(E, E_{3}, E_{\alpha \alpha}, E_{\alpha \beta}\right)\left(E_{3}\right)^{-\eta} \\
\quad=\mathcal{A}\left(\sqrt{E / E_{3}}, \sqrt{E_{\alpha \alpha} / E_{3}}, \sqrt{E_{\alpha \beta} / E_{3}}, A\right),
\end{aligned}
$$

where $\mathcal{O}$ is a general observable of the three-body system at energy $E$, with dimension of energy to the power $\eta$. In the present paper we discuss only the situation that we have only bound subsystems $(\alpha \alpha \beta)$; however, the analysis could be easily extended to other three-body halo systems such as the Borromean, tango, and $(\alpha \beta \alpha)$ systems.

In the case of the mean-square separation distances, $\left\langle r_{\gamma}^{2}\right\rangle$ with $\gamma=\alpha$ or $\beta$, i.e, the distance of the atom $\gamma$ to the c.m., and $\left\langle r_{\alpha \gamma}^{2}\right\rangle$, i.e, the distance between the atoms $\alpha$ and $\gamma$, the scaling functions are of the form

$$
\sqrt{\left\langle r_{\gamma}^{2}\right\rangle S_{3}}=\mathcal{R}_{\gamma}\left(\sqrt{\epsilon_{\alpha \alpha} / \epsilon_{3}}, \sqrt{\epsilon_{\alpha \beta} / \epsilon_{3}}, A\right)
$$

and

$$
\sqrt{\left\langle r_{\alpha \gamma}^{2}\right\rangle S_{3}}=\mathcal{R}_{\alpha \gamma}\left(\sqrt{\epsilon_{\alpha \alpha} / \epsilon_{3}}, \sqrt{\epsilon_{\alpha \beta} / \epsilon_{3}}, A\right),
$$

where $S_{3}$ is the smallest separation energy of the three-body system, i.e., $S_{3}=\min \left(E_{3}-E_{\alpha \alpha}, E_{3}-E_{\alpha \beta}\right)$. Two particular situations are worth mentioning, one is the case of trimer systems $(A=1)$, where the above scaling functions above reduce to

$$
\sqrt{\left\langle r_{\gamma}^{2}\right\rangle S_{3}}=\mathcal{R}_{\gamma}\left(\sqrt{\epsilon_{2} / \epsilon_{3}}\right)
$$

and

$$
\sqrt{\left\langle r_{\alpha \alpha}^{2}\right\rangle S_{3}}=\mathcal{R}_{\alpha \gamma}\left(\sqrt{\epsilon_{2} / \epsilon_{3}}\right) .
$$

The other special situation is found for $\epsilon_{\alpha \gamma}=0$ where the dimensionless product of the square radii and triatomic binding energy depend only on the mass ratio:

$$
\sqrt{\left\langle r_{\gamma}^{2}\right\rangle E_{3}}=\mathcal{R}_{\gamma}(A)
$$

and

$$
\sqrt{\left\langle r_{\alpha \gamma}^{2}\right\rangle E_{3}}=\mathcal{R}_{\alpha \gamma}(A)
$$

\section{Form factors}

The mean-square radii are calculated from the derivative of the Fourier transform of the respective matter density with respect to the square of the momentum transfer. The Fourier transform of the one- and two-body densities define the respective form factors, $F_{\beta}\left(q^{2}\right)$ and $F_{\alpha \gamma}\left(q^{2}\right)$, as a function of the dimensionless momentum transfer $\vec{q}$. For the meansquare radius of the particle $\gamma(=\alpha$ or $\beta$ ) to c.m., we have

$$
\left\langle r_{\gamma}^{2}\right\rangle=-6\left(1-\frac{m_{\gamma}}{2 m_{\alpha}+m_{\beta}}\right)^{2}\left|\frac{d F_{\gamma}\left(q^{2}\right)}{d q^{2}}\right|_{q^{2}=0},
$$

where

$$
\begin{aligned}
& F_{\alpha}\left(q^{2}\right)=\int d^{3} y d^{3} z \Psi_{\alpha \beta}\left(\vec{y}+\frac{\vec{q}}{2}, \vec{z}\right) \Psi_{\alpha \beta}\left(\vec{y}-\frac{\vec{q}}{2}, \vec{z}\right), \\
& F_{\beta}\left(q^{2}\right)=\int d^{3} y d^{3} z \Psi_{\alpha \alpha}\left(\vec{y}+\frac{\vec{q}}{2}, \vec{z}\right) \Psi_{\alpha \alpha}\left(\vec{y}-\frac{\vec{q}}{2}, \vec{z}\right) .
\end{aligned}
$$

For the mean-square distance between the particles $\alpha$ and $\gamma$, we have

$$
\left\langle r_{\alpha \gamma}^{2}\right\rangle=-\left.6 \frac{d F_{\alpha \gamma}\left(q^{2}\right)}{d q^{2}}\right|_{q^{2}=0},
$$

where

$$
F_{\alpha \gamma}\left(q^{2}\right)=\int d^{3} y d^{3} z \Psi_{\alpha \gamma}\left(\vec{y}, \vec{z}+\frac{\vec{q}}{2}\right) \Psi_{\alpha \gamma}\left(\vec{y}, \vec{z}-\frac{\vec{q}}{2}\right) .
$$

The above triatomic wave functions in momentum space are given in terms of the spectator functions $\chi_{\alpha \gamma}$ :

$$
\begin{aligned}
\Psi_{\alpha \alpha}(\vec{y}, \vec{z})= & \left(\frac{1}{\epsilon_{3}+\frac{A+2}{4 A} \vec{y}^{2}+\vec{z}^{2}}-\frac{1}{1+\frac{A+2}{4 A} \vec{y}^{2}+\vec{z}^{2}}\right) \\
& \times\left[\chi_{\alpha \alpha}(|\vec{y}|)+\chi_{\alpha \beta}\left(\left|\vec{z}-\frac{\vec{y}}{2}\right|\right)+\chi_{\alpha \beta}\left(\left|\vec{z}+\frac{\vec{y}}{2}\right|\right)\right],
\end{aligned}
$$

$$
\begin{aligned}
\Psi_{\alpha \beta}(\vec{y}, \vec{z})= & \left(\frac{1}{\epsilon_{3}+\frac{A+1}{2 A} \vec{z}^{2}+\frac{A+2}{2(A+1)} \vec{y}^{2}}\right. \\
& \left.-\frac{1}{1+\frac{A+1}{2 A} \vec{z}^{2}+\frac{A+2}{2(A+1)} \vec{y}^{2}}\right) \\
& \times\left[\chi_{\alpha \alpha}\left(\left|\vec{z}-\frac{A \vec{y}}{A+1}\right|\right)\right. \\
& \left.+\chi_{\alpha \beta}(|\vec{y}|)+\chi_{\alpha \beta}\left(\left|\vec{z}+\frac{\vec{y}}{A+1}\right|\right)\right],
\end{aligned}
$$

where $\vec{z}$ is the relative momentum of the pair and $\vec{y}$ is the relative momentum of the spectator particle to the pair in units of $\mu_{(3)}=1$. Note that the subindices of $\Psi$ in Eq. (18) 


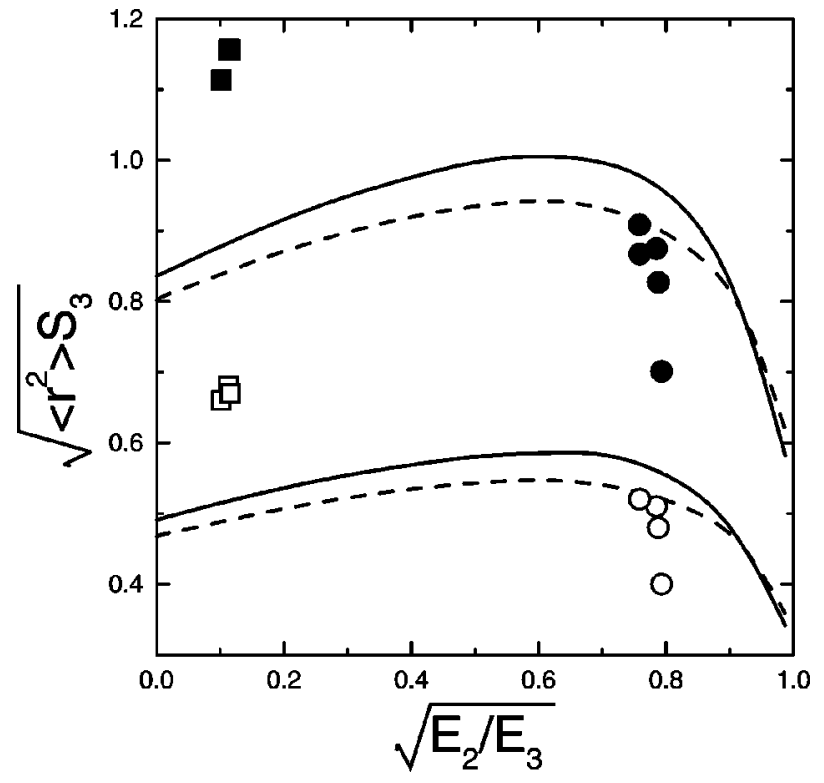

FIG. 1. The dimensionless products $\sqrt{\left\langle r_{\alpha}^{2}\right\rangle S_{3}}$ (lower curves) and $\sqrt{\left\langle r_{\alpha \alpha}^{2}\right\rangle S_{3}}$ (upper curves) as functions of $\sqrt{E_{2} / E_{3}}$. Our results for the ground state and first excited state are shown, respectively, by solid and dashed lines. Realistic calculations from Ref. [11], for $\sqrt{\left\langle r_{\alpha}^{2}\right\rangle S_{3}}$, are given by empty squares (ground state) and empty circles (excited state), and, for $\sqrt{\left\langle r_{\alpha \alpha}^{2}\right\rangle S_{3}}$, by full squares (ground state) and full circles (excited state).

just denote the pair of Jacobi relative momenta used to evaluate the wave function. For $\alpha \gamma$ with $\gamma=\alpha$ or $\beta$, one has the relative momentum between $\alpha$ and $\gamma$ and the relative momentum of the third particle to the center of mass of the system $\alpha \gamma$.

\section{RESULTS FOR TRIATOMIC RADII}

Our analysis has considered some particular three-body molecular systems, in which the three-body ground-state energy and the corresponding energies of the two-body subsystem are known theoretically for ${ }^{4} \mathrm{He}$ trimer [11], ${ }^{4} \mathrm{He}_{2}$ ${ }^{-6} \mathrm{Li},{ }^{4} \mathrm{He}_{2}-{ }^{7} \mathrm{Li}$, and ${ }^{4} \mathrm{He}_{2}-{ }^{23} \mathrm{Na}$ [23]. In Ref. [11], the authors have considered realistic two-body interactions; their results for the ground and excited state radii are appropriate for our purpose of comparing with the present scaling approach.

The ground and excited Efimov state energies of the ${ }^{4} \mathrm{He}_{3}$ molecule were extensively studied in the scaling approach of Refs. $[15,18]$, with the results that are in very good agreement with realistic calculations. This leads us to conclude that other details (beyond the dimer and trimer ground-state energies) presented in the realistic interactions, which have been used, are quite irrelevant to the existence of Efimov states. These features validates a universal scaling function, relating the trimer ground state, the dimer, and the weakly bound excited three-body energy state. Realistic calculations for the excited states of ${ }^{4} \mathrm{He}$ trimer approaches reasonably well the scaling limit $[15,18]$, which suggests to investigate the scaling limit of other observables such as the different radii defined in Eqs. (14) and (16). The conditions for the

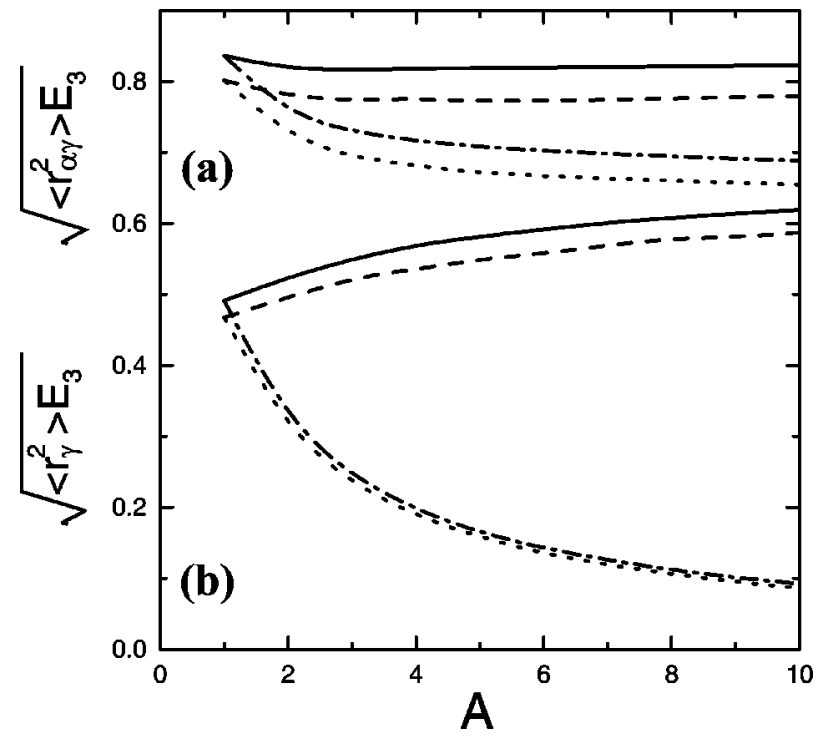

FIG. 2. For the triatomic $\alpha \alpha \beta$ system, with $\gamma \equiv \alpha, \beta$, it is shown the dimensionless products $\sqrt{\left\langle r_{\alpha \gamma}^{2}\right\rangle E_{3}}$ [upper (a) plots] and $\sqrt{\left\langle r_{\gamma}^{2}\right\rangle E_{3}}$ [lower (b) plots], as functions of $A \equiv m_{\beta} / m_{\alpha}$, in the limit $E_{\alpha \alpha}=E_{\alpha \beta}=0 . \sqrt{\left\langle r_{\gamma}^{2}\right\rangle}$ is the root-mean-square distance of particle $\gamma$ from the center of mass, and $\sqrt{\left\langle r_{\alpha \gamma}^{2}\right\rangle}$ is the root-mean-square distance between the particles $\alpha$ and $\gamma$. The results for the ground state $(N=0)$ are shown by the solid line $(\gamma=\alpha)$ and dot-dashed line $(\gamma=\beta)$, and, for the excited state $(N=1)$, by the dashed line $(\gamma=\alpha)$ and dotted line $(\gamma=\beta)$.

validity of the present approach are that the atoms should have a very shallow and short-ranged two-body interaction and the binding energy close to 0 , i.e., the ratio between the interaction range and dimer size should be much smaller than 1 . These are indeed the cases we are considering.

The results for the radii of ${ }^{4} \mathrm{He}_{3}$ molecule in the ground and excited states are shown in Fig. 1, in the form of a scaling plot. The dimensionless products $\sqrt{\left\langle r_{\alpha}^{2}\right\rangle S_{3}}$ and $\sqrt{\left\langle r_{\alpha \alpha}^{2}\right\rangle S_{3}}$ as functions of $\sqrt{E_{2} / E_{3}}$ are shown in the figure and compared to the realistic calculations, obtained from Ref. [11]. Our calculations for the ground and excited states are practically the same, which would be the case if the energies with respect to $\mu^{2}$ are, in fact, going to 0 , i.e., the scaling limit. The results for $\sqrt{\left\langle r_{\alpha}^{2}\right\rangle S_{3}}$ and $\sqrt{\left\langle r_{\alpha \alpha}^{2}\right\rangle S_{3}}$ for the excited state are in good agreement with the realistic result. However, for the ground state the results show a deviation of about $20 \%$. The excited state size is about ten times larger than the corresponding size of the ground state. Therefore, the scaling limit is better approached in the excited state, which is much larger than the interaction range, which is not strictly valid for the ground state, and consequently deviations in the scaling plot are stronger for this state.

In Fig. 2, the results for the dimensionless products $\sqrt{\left\langle r_{\alpha \alpha}^{2}\right\rangle E_{3}}, \sqrt{\left\langle r_{\alpha \beta}^{2}\right\rangle E_{3}}$, and $\sqrt{\left\langle r_{\gamma}^{2}\right\rangle E_{3}}$, as functions of $A$ $=m_{\beta} / m_{\alpha}$, for $E_{\alpha \alpha}=E_{\alpha \beta}=0$ are shown. We perform calculations for the ground $(N=0)$ and excited $(N=1)$ states, as indicated in the figure. One observes in the upper frame of Fig. 2 that the results almost saturate above $A \approx 3$ to the values found in the limit of $A=\infty$. The calculations for $A$ $=\infty$ give for $\left\langle r_{\alpha \alpha}^{2}\right\rangle$ the values of $0.69 / E_{3}$ for $N=0$ and 
TABLE I. Results for different radii of the molecular systems $\alpha \alpha \beta$, where $\alpha \equiv{ }^{4} \mathrm{He}$ and $\beta$ is identified in the first column. The ground-state energies of the triatomic molecules and the corresponding energies of the diatomic subsystems, obtained from Ref. [23], are given in the second, third, and fourth columns. $\left\langle r_{\alpha \gamma}^{2}\right\rangle$ is the corresponding mean-square distance between the particles $\alpha$ and $\gamma$ $(=\alpha, \beta) .\left\langle r_{\gamma}^{2}\right\rangle$ is the mean-square distance of $\gamma$ from the trimer center of mass.

\begin{tabular}{lccccccc}
\hline \hline & $\begin{array}{c}E_{3}^{(0)} \\
\beta\end{array}$ & $\begin{array}{c}E_{\alpha \alpha} \\
(\mathrm{mK})\end{array}$ & $\begin{array}{c}E_{\alpha \beta} \\
(\mathrm{mK})\end{array}$ & $\begin{array}{c}\sqrt{\left\langle r_{\alpha \alpha}^{2}\right\rangle} \\
(\AA)\end{array}$ & $\begin{array}{c}\sqrt{\left\langle r_{\alpha \beta}^{2}\right\rangle} \\
(\AA)\end{array}$ & $\begin{array}{c}\sqrt{\left\langle r_{\alpha}^{2}\right\rangle}(\AA) \\
(\AA)\end{array}$ & $\begin{array}{c}\sqrt{\left\langle r_{\beta}^{2}\right\rangle} \\
(\AA)\end{array}$ \\
\hline${ }^{4} \mathrm{He}$ & 106.0 & 1.31 & 1.31 & 9.45 & 9.45 & 5.55 & 5.55 \\
${ }^{6} \mathrm{Li}$ & 31.4 & 1.31 & 0.12 & 16.91 & 16.38 & 10.50 & 8.14 \\
${ }^{7} \mathrm{Li}$ & 45.7 & 1.31 & 2.16 & 14.94 & 13.88 & 9.34 & 6.31 \\
${ }^{23} \mathrm{Na}$ & 103.1 & 1.31 & 28.98 & 11.66 & 9.54 & 8.12 & 1.94 \\
\hline \hline
\end{tabular}

$0.61 / E_{3}$ for $N=1$. Therefore, for the ground state, the rootmean-square distance between two ${ }^{4} \mathrm{He}$ in the triatomic molecules can be estimated by $0.83 \sqrt{\hbar^{2} /\left(E_{3}{ }^{m_{4}} \mathrm{He}\right)}$, in the limit of zero pairwise binding energy. Our results for $\left\langle r_{\alpha \beta}^{2}\right\rangle$ are $0.45 / E_{3}$ for $N=0$ and $0.40 / E_{3}$ for $N=1$. The saturation value for $\sqrt{\left\langle r_{\alpha \alpha}^{2}\right\rangle E_{3}}$ is achieved fast with increasing $A$ than for $\sqrt{\left\langle r_{\alpha \beta}^{2}\right\rangle E_{3}}$, which depends on the difference in the masses of the atomic pair. The mean-square distance of one of the atoms $\gamma(=\alpha$ or $\beta)$ to the center of mass of the molecule can be obtained from the lower frame of Fig. 2, where we plot $\sqrt{\left\langle r_{\gamma}^{2}\right\rangle E_{3}}$ as a function of $A$. One sees that, for the infinitely heavy $\beta$ atom, the results for $\left\langle r_{\alpha}^{2}\right\rangle$ are the same as for $\left\langle r_{\alpha \beta}^{2}\right\rangle$, while $\sqrt{\left\langle r_{\beta}^{2}\right\rangle E_{3}}=0$, as the heavy particle should rest in the c.m. of the molecule in this limit. We remind the reader that the ratio between the binding energies for $N=0$ and $N=1$ is about 500 for $A=1$ [9] and 300 for $A=10$, while the dimensionless products of square radius and energy change only around $10 \%$.

In the above, we have considered examples of molecules with two helium atoms. However, our results presented in Figs. 1 and 2 are more general, such that we can extend the estimates to other atomic systems. Of particular interest is the analysis of possible formation of molecular systems in experiments with ultracold trapped gases. By considering, for example, the estimates of trimer energies obtained from the recombination coefficient, given in Ref. [10], within our approach we can predict the corresponding trimer sizes. In this case, we have $\alpha=\beta$ and $A=1$ in the previous equations and in the figures. However, our unit for a specific trimer of an atom with $A$ nucleons will be $\hbar^{2} / m_{A}=(48.48 / A) \mathrm{K} \AA^{2}$.

In Table I, we present our results for the different radii of the ground state $(N=0)$ of the weakly bound molecular systems $\alpha \alpha \beta$, where $\alpha \equiv{ }^{4} \mathrm{He}$ and $\beta={ }^{4} \mathrm{He},{ }^{6} \mathrm{Li},{ }^{7} \mathrm{Li}$, and ${ }^{23} \mathrm{Na}$, obtained from the known theoretical values of $E_{3}^{(0)}, E_{\alpha \alpha}$, and $E_{\alpha \beta}$ [23]. Our calculation for ${ }^{4} \mathrm{He}_{3}$ of $\sqrt{\left\langle r_{\alpha \alpha}^{2}\right\rangle}$ gives $9.45 \AA$, which is $14 \%$ of the value $11 \AA$ obtained in the realistic variational calculations of Ref. [11]. The same quality of agreement is found for $\sqrt{\left\langle r_{\alpha}^{2}\right\rangle}$, which in our calculation is $5.55 \AA$ compared to $6.4 \AA$ of Ref. [11]. The quality of the reproduction of the realistic results by our calculations are quite surprising in view of the simplicity of the present ap-
TABLE II. Results for the size of trimer systems predicted in Ref. [10]. The sizes are given by $\sqrt{\left\langle r_{\alpha}^{2}\right\rangle}$, the root-mean-square distance between the atom $\alpha$ and the center of mass of the trimer system. The atoms of the trimer are identified in the first column. For each dimer energy, given in the second column, we have two possible trimer energies (columns 3 and 5), with the corresponding radii given in columns 4 and 6 .

\begin{tabular}{|c|c|c|c|c|c|}
\hline Atom & $\begin{array}{c}E_{2} \\
(\mathrm{mK})\end{array}$ & $\begin{array}{c}E_{3} \\
(\mathrm{mK})\end{array}$ & $\begin{array}{c}\sqrt{\left\langle r_{\alpha}^{2}\right\rangle} \\
(\AA)\end{array}$ & $\begin{array}{c}E_{3}^{\prime} \\
(\mathrm{mK})\end{array}$ & $\begin{array}{c}\sqrt{\left\langle r_{\alpha}^{2}\right\rangle^{\prime}} \\
(\AA)\end{array}$ \\
\hline${ }^{23} \mathrm{Na}|1,-1\rangle$ & 2.85 & 7.75 & 12 & 3.06 & 38 \\
\hline${ }^{87} \mathrm{Rb}|1,-1\rangle{ }^{\mathrm{a}}$ & 0.17 & 0.56 & 22 & 0.175 & 114 \\
\hline${ }^{87} \mathrm{Rb}|1,-1\rangle^{\mathrm{b}}$ & 0.17 & 0.47 & 25 & 0.183 & 91 \\
\hline${ }^{85} \mathrm{Rb}|2,-2\rangle$ & $1.3 \times 10^{-4}$ & $2.4 \times 10^{-4}$ & 1293 & $1.7 \times 10^{-4}$ & 1944 \\
\hline
\end{tabular}

${ }^{\mathrm{a}}$ The trimer estimates, given in Ref. [10], for ${ }^{87} \mathrm{Rb}|1,-1\rangle$, are for noncondensed trapped atoms.

${ }^{\mathrm{b}}$ The trimer estimates, given in Ref. [10], for ${ }^{87} \mathrm{Rb}|1,-1\rangle$, are for condensed trapped atoms.

proach, where the only physical inputs are the values of the dimer and trimer binding energies. The several different radii of the molecules ${ }^{4} \mathrm{He}_{2}-{ }^{6} \mathrm{Li},{ }^{4} \mathrm{He}_{2}{ }^{7} \mathrm{Li}$, and ${ }^{4} \mathrm{He}_{2}-{ }^{23} \mathrm{Na}$ have values larger than those found in the ${ }^{4} \mathrm{He}_{3}$, which makes plausible that our predictions are even better in quality.

We point out that the results in Table I for the ${ }^{4} \mathrm{He}$ dimer sizes inside the molecules shrink with respect to the free value of $52 \AA$, due to the large values of the trimer binding energies. Qualitatively this is explained just by considering that the dimer size scales roughly with the inverse of the square-root of its binding energy inside the molecule, which can be estimated to be two-thirds of the molecule binding, from which one finds for that the dimer has sizes around $10 \AA$, close to that we have found in Table I.

In Table II, we are also presenting results for different radii of the trimers predicted in Ref. [10], from where we obtain the energy of the dimer and the most weakly bound trimer energies of ${ }^{23} \mathrm{Na}\left|F=1, m_{F}=-1\right\rangle,{ }^{87} \mathrm{Rb} \mid F=1, m_{F}$ $=-1\rangle$, and ${ }^{85} \mathrm{Rb}\left|F=2, m_{F}=-2\right\rangle$, where $\left|F, m_{F}\right\rangle$ is the respective hyperfine states of the total spin $F$. We are presenting the mean-square distance from each atom to the center of mass of the corresponding trimer. From Fig. 1, one can also obtain the corresponding mean-square distance between the atoms. We observe that one value of the recombination rate is consistent with two values of the most weakly bound trimer energy, as discussed in Ref. [10]. Therefore, we present two possible values for the radii that are consistent with the corresponding weakly bound trimer energies. Actually, we should also mention that, in a trap, one can achieve dimer and trimer molecules with very large sizes, following the possibility to alter the corresponding two-body scattering length [5].

Finally, it is interesting to recall the results for the hyperradius calculations obtained by Jensen and collaborators [21]. From their scaling plot, one can observe that the hyperradius of a tango system is bigger than the hyperradius of a Borromean system, for the same three-body energy. This point can become very clear, for instance, if we take as an example the results shown for the ${ }_{\Lambda}^{3} H$ (filled circles in Fig. 2 
of Ref. [21]) and estimate the dimensionless product of observables, $\left\langle\rho^{2}\right\rangle m B / \hbar^{2}$ (product of the $x$ axis and $y$ axis in Fig. 2 of Ref. [21], where $\rho$ is the hyperradius and $B$ is the three-body binding energy). When going from the tango to the Borromean configuration, this product will decrease. Therefore, if one keeps the same binding energy, the Borromean system would be more compact than the tango system. Extending this analysis to $(\alpha \beta \alpha)$ halos, where $(\alpha$ $-\alpha)$ is virtual and $(\alpha \beta)$ is bound, and also to all-bound pairs $(\alpha \alpha \beta)$, one should expect that the sizes increase when going from the Borromean states to halos with all-bound subsystems, while keeping the three-body energy fixed. Within our approach, the scaling relations are expected to be followed in all the cases, in the limit of a zero-range interaction. The Borromean halo (the less attractive system), in order to have the same three-body energy as a tango state, should be more compact in agreement with the scaling plot of Ref. [21]. In a realistic case, our scheme is expected to produce better results in the all-bound case, when comparing systems with the same three-body energies. This occurs because the all-bound case would have the most extended wave function; consequently, the range of the potential, in relation to the size, would have the smallest value, satisfying better the validity condition for the scaling relations, which is that the interaction range must be small compared to particle distances.

\section{CONCLUSIONS}

The mean-square radii of the triatomic molecules ${ }^{4} \mathrm{He}_{3}$, ${ }^{4} \mathrm{He}_{2}-{ }^{6} \mathrm{Li},{ }^{4} \mathrm{He}_{2}-{ }^{7} \mathrm{Li}$, and ${ }^{4} \mathrm{He}_{2}-{ }^{23} \mathrm{Na}$ are calculated using a renormalized three-body model with a pairwise Dirac- $\delta$ interaction, having as physical inputs only the values of the binding energies of the diatomic and triatomic molecules. Presently, we have considered molecular three-body systems with bound subsystems, due to the available data. The renormalized zero-range model can also be applied to the cases where at least one of the subsystems is virtual. When comparing systems with the same three-body energies and potential ranges, the validity of the renormalized zero-range model is expected to be better in the all-bound case, because this case corresponds to the most extended wave function.

The validity of the present framework is substantiated by the agreement of our results for the different radii with the realistic potential model calculations of Ref. [11] for ${ }^{4} \mathrm{He}_{3}$ ground and excited states, which are within about $14 \%$. These results are quite surprising in view of the simplicity of the approach, where only the physical inputs are the values of diatomic and triatomic binding energies. We predicted for the first time, as far as we know, the values of several different radii for ${ }^{4} \mathrm{He}_{2}-{ }^{6} \mathrm{Li},{ }^{4} \mathrm{He}_{2}-{ }^{7} \mathrm{Li}$, and ${ }^{4} \mathrm{He}_{2}-{ }^{23} \mathrm{Na}$ molecules, from the theoretical values of the binding energies calculated in Ref. [23]. These other molecules are, in general, larger than the ${ }^{4} \mathrm{He}$-trimer, indicating that our radii predictions for these triatomic ground states can be even better in quality than those found for ${ }^{4} \mathrm{He}_{3}$.

In view of the actual relevance of ultracold atomic systems that are being experimentally studied, and the possibility to observe the formation of molecular systems in trapped condensates, we also present results for the sizes of rubidium and sodium trimers, considering the binding energies that were recently estimated [10] from the analysis of the corresponding three-body recombination coefficients.

\section{ACKNOWLEDGMENTS}

We would like to thank Fundação de Amparo à Pesquisa do Estado de São Paulo (FAPESP) for partial support. L.T. and T.F. also thank Conselho Nacional de Desenvolvimento Científico e Tecnológico (CNPq) partial support.
[1] E. Nielsen, D.V. Fedorov, A.S. Jensen, E. Garrido, Phys. Rep. 347, 373 (2001).

[2] W. Schöllkopf and J.P. Toennies, Science 266, 1345 (1994); K.T. Tang, J.P. Toennies, and C.L. Liu, Phys. Rev. Lett. 74, 1546 (1995).

[3] R.E. Grisenti, W. Schöllkopf, J.P. Toennies, G.C. Hegerfeldt, T. Köhler, and M. Stoll, Phys. Rev. Lett. 85, 2284 (2000).

[4] M.H. Anderson, J.R. Ensher, M.R. Matthews, C.E. Wieman, and E.A. Cornell, Science 269, 198 (1995); C.C. Bradley, C.A. Sackett, J.J. Tollet, and R.G. Hulet, Phys. Rev. Lett. 75, 1687 (1995); K.B. Davis, M.-O. Mewes, M.R. Andrews, N.J. van Druten, D.S. Durfee, D.M. Kurn, and W. Ketterle, ibid. 75, 3969 (1995).

[5] S. Inouye, M.R. Andrews, J. Stenger, H.-J. Miesner, D.M. Stamper-Kurn, and W. Ketterle, Nature (London) 392, 151 (1998); E. Timmermans, P. Tommasini, M. Hussein, and A. Kerman, Phys. Rep. 315, 199 (1999).

[6] N.R. Claussen, E.A. Donley, S.T. Thompson, and C.E. Wieman, Phys. Rev. Lett. 89, 010401 (2002); E.A. Donley, N.R. Claussen, S.T. Thompson, and C.E. Wieman, Nature (London) 417, 529 (2002).
[7] R.H. Wynar, R.S. Freeland, D.J. Han, C. Ryu, and D.J. Heinzen, Science 287, 1016 (2000).

[8] C. Mckenzie et al., Phys. Rev. Lett. 88, 120403 (2001).

[9] V. Efimov, Phys. Lett. B 33, 563 (1970); Nucl. Phys. A 362, 45 (1981); V. Efimov, Comments Nucl. Part. Phys. 19, 271 (1990), and references therein.

[10] M. T. Yamashita, T. Frederico, A. Delfino, and L. Tomio, Phys. Rev. A (to be published), e-print cond-mat/0206317.

[11] P. Barletta and A. Kievsky, Phys. Rev. A 64, 042514 (2001).

[12] V. Roudnev and S. Yakovlev, Chem. Phys. Lett. 328, 97 (2000).

[13] S.K. Adhikari, T. Frederico, and I.D. Goldman, Phys. Rev. Lett. 74, 487 (1995); S.K. Adhikari and T. Frederico, ibid. 74, 4572 (1995).

[14] A.E.A. Amorim, L. Tomio, and T. Frederico, Phys. Rev. C 56, R2378 (1997).

[15] T. Frederico, A. Delfino, A.E.A. Amorim, and L. Tomio, Phys. Rev. A 60, R9 (1999).

[16] A. Delfino, T. Frederico, and L. Tomio, Few-Body Syst. 28, 259 (2000); J. Chem. Phys. 113, 7874 (2000). 
[17] L.H. Thomas, Phys. Rev. 47, 903 (1935).

[18] M.T. Yamashita, T. Frederico, A. Delfino, and L. Tomio, Phys. Rev. A 66, 052702 (2002).

[19] M.V. Zhukov, B.V. Danilin, D.V. Fedorov, J.M. Bang, I.J. Thompson, and J. Vaagen, Phys. Rep. 231, 151 (1993).

[20] F. Robichaux, Phys. Rev. A 60, 1706 (1999).
[21] A.S. Jensen, K. Riisager, D.V. Fedorov, and E. Garrido, Europhys. Lett. 61, 320 (2003).

[22] T. Frederico and I.D. Goldman, Phys. Rev. C 36, R1661 (1987).

[23] J. Yuan and C.D. Lin, J. Phys. B 31, L637 (1998). 